\title{
ARTICLE OPEN Realizing Haldane model in Fe-based honeycomb ferromagnetic insulators
}

\author{
Heung-Sik Kim ${ }^{1}$ and Hae-Young Kee ${ }^{1,2}$
}

The topological Haldane model on a honeycomb lattice is a prototype of systems hosting topological phases of matter without external fields. It is the simplest model exhibiting the quantum Hall effect without Landau levels, which motivated theoretical and experimental explorations of topological insulators and superconductors. Despite its simplicity, its realization in condensed matter systems has been elusive due to a seemingly difficult condition of spinless fermions with sublattice-dependent magnetic flux terms. While there have been theoretical proposals including elaborate atomic-scale engineering, identifying candidate topological Haldane model materials has not been successful, and the first experimental realization was recently made in ultracold atoms. Here, we suggest that a series of Fe-based honeycomb ferromagnetic insulators, $A \mathrm{Fe}_{2}\left(\mathrm{PO}_{4}\right)_{2}(A=\mathrm{Ba}, \mathrm{Cs}, \mathrm{K}, \mathrm{La})$ possess Chern bands described by the topological Haldane model. How to detect the quantum anomalous Hall effect is also discussed.

npj Quantum Materials (2017)2:20; doi:10.1038/s41535-017-0021-z

\section{INTRODUCTION}

In 1988, F.D.M. Haldane introduced an idea of the quantum Hall effect without Landau levels, and a simple tight-binding model of spinless fermions on a honeycomb lattice was suggested as an example, ${ }^{1}$ which was dubbed the topological Haldane model (THM). It features a chiral edge spectrum with a Chern number without external magnetic field, which is a prototype of the quantum anomalous hall (QAH) insulator. ${ }^{2-4}$ Although THM was "unlikely to be directly physically realizable", as Haldane stated in his paper, ${ }^{1}$ yet his vision of intrinsic topological state of matter in condensed matter systems inspired later discoveries of timereversal symmetric topological insulators ( $\mathrm{TI})$ and promoted other topological phases. ${ }^{5-7}$

The THM is a spinless fermion model in a honeycomb lattice with nearest neigbor (n.n.) and complex next nearest neighbor (n.n.n.) hopping integrals: ${ }^{1}$

$H=t_{1} \sum_{\langle i j\rangle} c_{i}^{\dagger} c_{j}+t_{2} \sum_{\langle\langle i j\rangle\rangle} e^{i \Phi_{i j}} c_{i}^{\dagger} c_{j}+$ h.c.

where $t_{1}$ and $t_{2}$ are real and represent n.n. and n.n.n. hopping integral terms, respectively. $\Phi_{i j}$ breaks time-reversal symmetry (TRS), and its sign differs for two sublattices $(A, B)$, i.e., $\Phi$ for $A$ and $-\Phi$ for $B$. Realization of the THM requires spinless fermions hopping on honeycomb lattice with spatially alternating flux yielding Aharonov-Bohm phase $i \Phi_{i j}$.

Due to these difficult requirements of the THM, realization of $\mathrm{QAH}$ effect in materials was achieved only after the discovery of quantum spin hall (QSH) insulator. Since each spin component of electrons in QSH insulators is regarded as a QAH state, one can obtain a QAH effect if one spin component QAH state is removed. The discovery of $\mathrm{TI}^{8}, 9$ together with recent advancement of atomic-scale engineering techniques, then revived the interest for the QAH phase. There have been a surge of theoretical proposals in various system including magnetic-ion-doped $\mathrm{HgTe}$ quantum well $^{10}$ and TI surfaces, ${ }^{11}$ engineered graphene, ${ }^{12,}{ }^{13}$ transition metal oxides ${ }^{14,15}$ and their heterostructures. ${ }^{16,}{ }^{17}$ On the other hand, experimental observations of QAH effect was reported only in $\mathrm{Cr}$ - and $\mathrm{V}$-doped $(\mathrm{Bi}, \mathrm{Sb})_{2} \mathrm{Te}_{3}$ film, ${ }^{18,19}$ confirming the idea that $\mathrm{a}$ $\mathrm{TI}$ with magnetic impurities removes one spin QAH state and reveals the QAH effect. Alternatively, the idea of breaking TRS by exerting circularly polarized ac-electric field and inducing $\mathrm{QAH}$ phase was suggested in light of the Floquet-Bloch theory ${ }^{20,21}$ and has been realized recently. ${ }^{22,}{ }^{23}$ Also, the experimental realization of the THM was recently made in ultracold atomic fermions in a periodically modulated honeycomb lattice. ${ }^{24}$ However, it seems that realizing the THM in a simple two-dimensional (2D) honeycomb compounds in an equilibrium situation becomes at a glance an unrealistic task.

Here we show that the THM, original QAH model can be found in Fe-based honeycomb ferromagnetic insulators. With the help of strong Hund's coupling in Fe, electrons with one major spincomponent (say down-spin) are fully polarized in occupied bands. Then electrons with other spin component (up-spin) form Haldane bands with finite Chern numbers, described by effective spinless fermions with complex n.n.n. hoppings. We find that a series of Febased honeycomb stoichiometric materials, $\mathrm{AFe}_{2}\left(\mathrm{PO}_{4}\right)_{2}(\mathrm{AFPO}$, $A=\mathrm{Ba}, \mathrm{K}, \mathrm{Cs}, \mathrm{La})$, fall into a class of these materials described by the THM. Among them, compounds with $A=\mathrm{K}, \mathrm{Cs}$, and La exhibit a $\mathrm{QAH}$ effect, while $\mathrm{BaFe}_{2}\left(\mathrm{PO}_{4}\right)_{2}$ does not show a QAH effect, because two Chern bands have opposite chirality.

$\mathrm{BaFe}_{2}\left(\mathrm{PO}_{4}\right)_{2}$ (BFPO), a recently synthesized insulator, is the first example of $2 \mathrm{D}$ ising ferromagnetic oxides, where honeycomb layers are made of $\mathrm{FeO}_{6}$ octahedra (naming oxides is due to $\mathrm{FeO}_{6}$ octahedra forming honeycomb lattice). Ferromagnetic transition occurs at $T_{\mathrm{c}} \sim 65 \mathrm{~K}$, and $\mathrm{Fe}^{2+}\left(d^{6}\right)$ high-spin moments on honeycomb lattices align along the layer-normal direction below $T_{c .}{ }^{25}$ Interestingly, it also shows an intriguing re-entrant structural transition at $T_{\mathrm{c}}$ from monoclinic $P \overline{1}$ to rhombohedral $R \overline{3}$

\footnotetext{
'Department of Physics, University of Toronto, Toronto, ON M5S 1A7, Canada and ${ }^{2}$ Canadian Institute for Advanced Research/Quantum Materials Program, Toronto, ON MSG 1Z8, Canada

Correspondence: Hae-Young Kee (hykee@physics.utoronto.ca)
}

Received: 7 November 2016 Revised: 13 February 2017 Accepted: 23 February 2017

Published online: 03 April 2017 
symmetries with most likely due to the coupling between ferromagnetic ordering and lattice structure via spin-orbit coupling (SOC). Signature of orbital angular momentum of $1 \mu_{\mathrm{B}}$ at Fe site was reported, implying significant role of atomic SOC in BFPO. ${ }^{26}$ It was suggested that electronic correlation turns the system from a semimetal to a Mott insulator with Fe atomic orbital angular momentum. ${ }^{27}$ We find that the atomic orbital momentum in BFPO signals possible Haldane bands via combined effects of Coulomb interactions and SOC under the ferromagnetic order. Two copies of Haldane Chern bands are identified; one set of Chern bands just above and the other set of Chern bands below the Fermi level, with opposite chirality of the first set. Thus, BFPO is a trivial ferromagnetic Mott insulator. However, this provides us a guideline to identify the Haldane Chern insulators, because Haldane Chern insulators described by the THM with effective hopping integrals can be achieved by adding or subtracting an electron per formula unit. Substituting $\mathrm{Ba}$ into $\mathrm{Cs}, \mathrm{K}$, or La leads to new compounds $\mathrm{CsFe}_{2}\left(\mathrm{PO}_{4}\right)_{2}$ (CFPO), $\mathrm{KFe}_{2}\left(\mathrm{PO}_{4}\right)_{2} \quad(\mathrm{KFPO})$, or $\mathrm{LaFe}_{2}\left(\mathrm{PO}_{4}\right)_{2}$ (LFPO). We find that the structural derivatives have stable layered honeycomb structures, and exhibit nontrivial bulk Chern numbers, featured by chiral surface states and a bulk gap of at least $\sim 0.2 \mathrm{eV}$, in the presence of Coulomb interactions of Fe $d$ orbitals.

\section{RESULTS}

Figure 1 shows the evolution of Fe $d$-orbital in BFPO with respect to inclusions of relevant energy scales. In a $3 d^{6}$ configuration in the atomic limit, the dominant energy scale is the exchange splitting between different spin components introduced by the Hund's coupling. Density functional theory (DFT) calculation yields the energy difference of $5 J_{H} \sim 3 \mathrm{eV}$ between the down and up spin states, ${ }^{27}$ which is much larger than the cubic crystal field of $\sim 1 \mathrm{eV}$ exerted by an oxygen octahedral cage surrounding Fe. Hence five electrons with down-spin occupy the bands well below the Fermi level, and only one electron with up spin is left in the $t_{2 g}$ minor spin states, which acts as spinless fermion. The system without SOC becomes half-metallic when no further on-site energy scales are included, as shown in Fig. 1. The presence of trigonal distortion in $\mathrm{FeO}_{6}$ octahedra further splits the $t_{2 g}$ states into $a_{1 \mathrm{~g}}$ singlet and $e^{\prime}{ }_{\mathrm{g}}$ doublet;

$$
\begin{aligned}
& \left|e^{\prime}{ }_{g} \pm\right\rangle \equiv \frac{1}{\sqrt{3}}\left(\left|d_{x y}\right\rangle+e^{ \pm i \theta}\left|d_{y z}\right\rangle+e^{\mp i \theta}\left|d_{x z}\right\rangle\right), \\
& \left|a_{1 g}\right\rangle \equiv \frac{1}{\sqrt{3}}\left(\left|d_{x y}\right\rangle+\left|d_{y z}\right\rangle+\left|d_{x z}\right\rangle\right),
\end{aligned}
$$

where $\theta=2 \pi / 3$. Note that, the $t_{2 g}$ triplet corresponds to an effective atomic orbital angular momentum $l^{\text {eff }}=1$ complex, $a_{1 \mathrm{~g}}$ and $e^{\prime}{ }_{g \pm}$ to $I_{\mathrm{n}}^{\text {eff }}=0$ and \pm 1 eigenstates, respectively, where $\mathbf{n}$ is the layer-normal direction. Finally, in the presence of ferromagnetic order parallel to $\mathbf{n}$, as reported in BFPO, the spin degree of freedom is frozen and SOC takes the form of $\lambda \hat{l}_{n}^{\text {eff }}\left\langle S_{n}\right\rangle$, where the positive $\lambda$ is the SOC magnitude of Fe $d$-orbital, $\hat{l}_{n}^{\text {eff }}$ is the effective atomic orbital angular momentum operator along $\mathbf{n}$, and $\left\langle S_{n}\right\rangle$ is magnitude of the FM order. Hence SOC under the FM order behaves as an atomic orbital Zeeman field to the $e^{\prime}{ }_{g \pm}$ states making $e^{\prime}{ }_{g-}$ state lower in energy than $e^{\prime}{ }_{g \pm}$. Note that, without SOC these two atomic orbital states are degenerate, and the degeneracy is protected at $\Gamma$ and $K$ points even after hopping integrals are introduced to form the Bloch bands. The gap at $\Gamma$ and $K$ opens up when SOC is introduced.

The on-site Coulomb interaction enhances the gap between the $e_{g_{+}}^{\prime}$ and $e^{\prime}{ }_{g-}$ Bloch states and gets bigger by the interaction parameter $U$. Due to the strong $d$-orbital Coulomb interaction in Fe, the system fully polarizes $e^{\prime}{ }_{g \pm}$ orbitals, i.e., the both up spin $e_{g}^{\prime}$ - and $e^{\prime}{ }_{g_{+}}$orbitals are fully occupied and empty, respectively, as reported earlier in ref. 27 . Since the effective and real atomic orbital momenta are antiparallel to each other, the spin and the real orbital momenta at Fe add up to yield total magnetic moment of $\sim 5 \mu_{\mathrm{B}}$, much larger than the size of $d^{6}$ high-spin moment $\mathrm{S}=2 \mu_{\mathrm{B}}$ (assuming the $g$-factor $\sim 2$ ). This is consistent to the value reported in experiment, ${ }^{26}$ which confirms that the Hubbard $U_{\text {eff }}$ should be larger than $3 \mathrm{eV}$, above which size of the total magnetic moment saturates close to the observed one.

Let us now construct an effective tight-binding model consisting of the $e^{\prime}{ }_{g_{+}}$orbital. Figure 2 shows the schematic shape of $e^{\prime}{ }_{g_{+}}$ orbital. First, n.n. hopping terms between the $e^{\prime}{ }_{g+}$ orbitals are realvalued due to the presence of n.n. inversion centers enforcing cancellation of complex phases. On the other hand, n.n.n. hopping channels can have complex values since they do not have any symmetry constraint. For example, let us consider one n.n.n. hopping channel between the $e^{\prime}{ }_{g_{+}}$orbitals at A-sublattice along the horizontal direction, as shown in lower figure in Fig. 2.

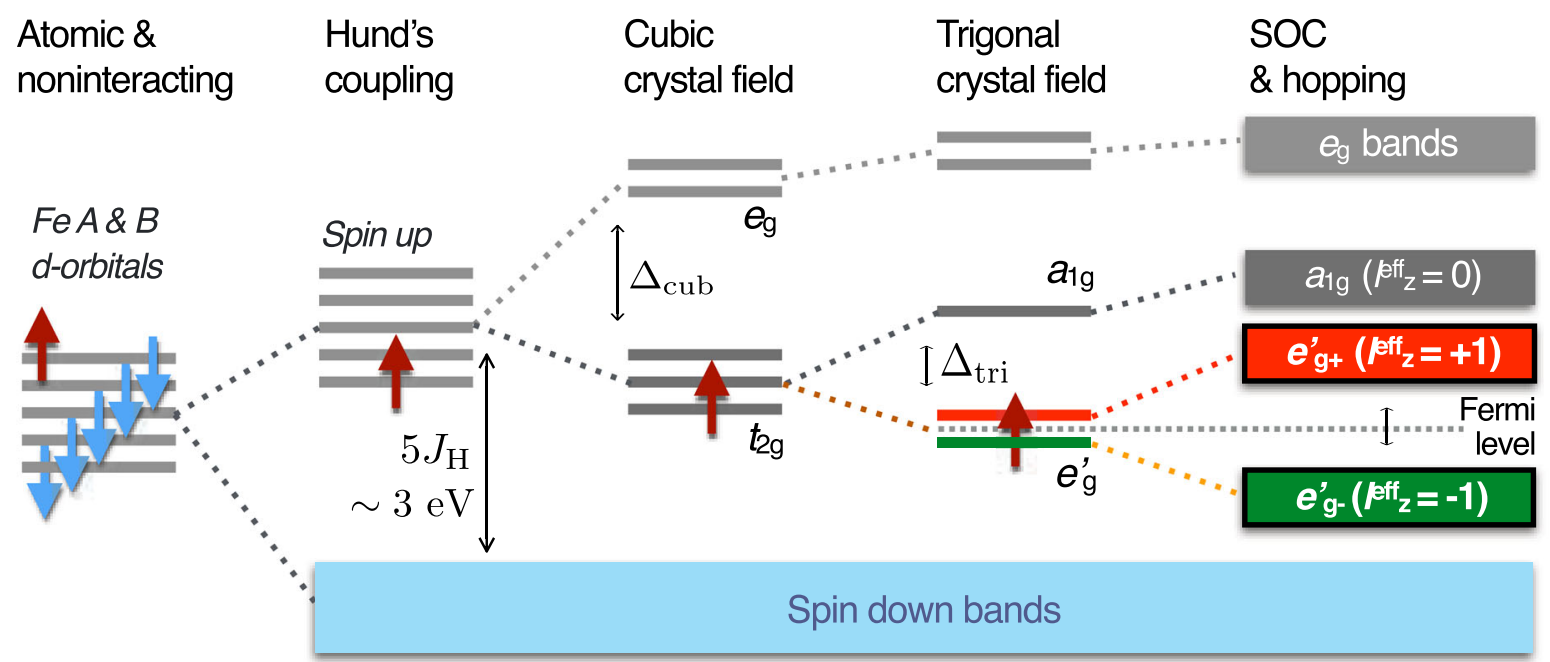

Fig. 1 Energy level diagram of $\mathrm{Fe}^{2+}$ in $\mathrm{BaFe}_{2}\left(\mathrm{PO}_{4}\right)_{2}$ (BFPO), with respect to inclusion of relevant energy scales $U>J_{\mathrm{H}}>\Delta_{\text {cub }}>\Delta_{\text {tri }} \gtrsim \lambda_{\text {so }} \approx t_{\text {hop }}$ 

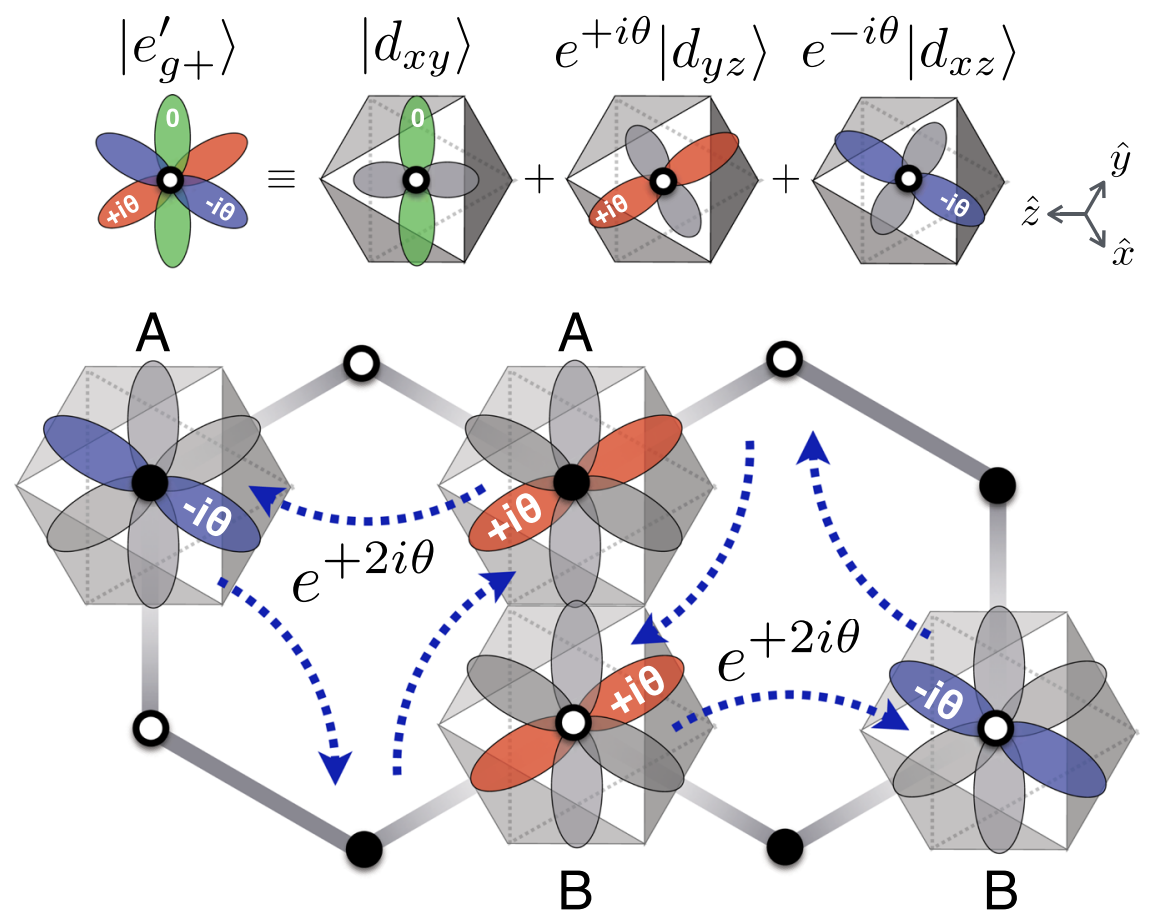

Fig. 2 Illustration of a orbital at Fe site and depiction of next-nearest-neighbor (n.n.n.) complex hopping terms between the $e^{\prime}{ }_{g \pm}$ orbitals. In the hopping figure, $d x z, y z$ orbitals, which contribute most to the horizontal n.n.n. hopping, are colored within each $e^{\prime}{ }_{ \pm \pm}$state. Note that, the $n$. n.n. hopping channels represented as dashed blue arrows can be transformed to each other by the inversion at the n.n. bond center and the threefold rotation symmetries (color online)

Assuming only one off-diagonal hopping channel between the $\mathrm{n}$. n.n. $t_{2 g}$ orbitals is active (between $d_{y z}$ and $d_{x z}$ orbitals, highlighted in blue and red colors in Fig. 2 , respectively), in the $e^{\prime}{ }_{g+}$ subspace it yields complex hopping term $t_{2} e^{+2 i \theta}$, where the phase $2 \theta$ originates from $e^{ \pm i \theta}$ assigned to $d_{y z}$ and $d_{x z}$ orbitals, respectively. The presence of the $n . n$. inversion centers and additional threefold symmetry then generates all other n.n.n. channels; $t_{2} e^{+2 i \theta}$ term for both $A$ and $B$ sublattices in a counterclockwise direction as shown in Fig. 2. For $e^{\prime}{ }_{g-}$ orbitals, on the other hand, $t_{2} e^{-2 i \theta}$ terms for counterclockwise n.n.n. hopping channels are obtained. The complex phase can deviate from $2 \theta$ depending on details of the $t_{2 g}$ hopping channels, but in general it does not vanish. Hence all of the conditions for realizing THM are fulfilled in BFPO, so that the system consists of two sets of THM $\left(e^{\prime}{ }_{g^{-}}\right.$and $\left.e^{\prime}{ }_{g_{-}}-\mathrm{THM}\right)$ with opposite chiralities to each other. As shown in Fig. 1, the $e_{g-}^{\prime}$ bands are fully occupied while $e^{\prime}{ }_{g^{+}}$bands are empty and mixed with $a_{1 \mathrm{~g}}$ band. Hence BFPO is in a trivial ferromagnetic Mott insulator phase, as the Fermi level lies in between two sets of Haldane bands with opposite Chern numbers.

The key ingredients for realizing the THM are spin-polarization due to strong Hund's coupling, and a separation of $e^{\prime}{ }_{g+}$ and $e^{\prime}{ }_{g-}$ orbitals introduced by the broken TRS and SOC, as described above. Then, within the $e_{g^{+}}^{\prime}$ (or $e_{g_{-}}^{\prime}$ ) subspace the phase factor of $e^{ \pm 2 i \theta}$ emerges from the complex nature of the orbital wavefunction. To confirm our arguments above, DFT calculations incorporating Coulomb interactions and SOC are carried out. DFT $+U^{28}$ and Heyd-Scuseria-Ernzerhof (HSE) hybrid functional formalisms ${ }^{29}$ are employed, which yield consistent results to each other when the value of $U_{\text {eff }} \equiv U-J$ parameter for Fe $d$-orbital in DFT $+U$ computations is about $4 \sim 5 \mathrm{eV}$ (in the weakly interacting regime $\left(U_{\text {eff }}<3 \mathrm{eV}\right)$ of BFPO, a narrow-gap Chern insulator/semimetal phase was reported previously in a DFT $+U$ study. The main focus of this study is how to realize the THM in the strongly interacting regime of $3<U_{\text {eff }}<6 \mathrm{eV}$ which is a realistic parameter range for Fe $d$-orbital. For the computational details see Supplementary
Material). ${ }^{30}$ Figure $3 a$ shows the $D F T+U\left(U_{\text {eff }}=3 \mathrm{eV}\right)$ bands for single-layer BFPO (SL-BFPO). First we comment that, while the bulk unit cell contains three $\mathrm{Fe}_{2}\left(\mathrm{PO}_{4}\right)_{2}$ layers, the band splitting due to interlayer coupling is negligibly small as shown in Supplementary Material. This manifests the quasi-2D electronic structure of this compound, possibly one of the closest to the 2D limit among the other quasi-2D layered compounds ever synthesized. Hence in Fig. 3 SL-AFPO bands are shown. The spin is oriented along the $\mathbf{n}$ direction, and all the down spin states are located below $-2 \mathrm{eV}$, as shown in the projected density of states plot. About $1.5 \mathrm{eV}$ below and $2 \mathrm{eV}$ above the Fermi level, the up spin $e_{\mathrm{g}-}$ and $e_{\mathrm{g}+}$ bands, respectively, are located. Chern numbers for the $e_{\mathrm{g}-}$ bands are \pm 1 (for bulk \pm 3 , with each layer contributing \pm 1 ).

While BFPO is a failed QAH though described by the THM, this system offers us an insight. One can obtain a half-filled $e_{\mathrm{g} \pm}^{\prime}$ bands by adding one electron or hole per a formula unit, and this can be achieved by substituting Ba into alkali or lanthanide elements of similar ionic radii with $\mathrm{Ba}^{2+}$, say $\mathrm{K}^{1+}$ and $\mathrm{La}^{3+}$ for alkali and rareearth ions, respectively (applying gate voltage on 2D BFPO sheets is another way to generate $\mathrm{QAH}$ effects and to reveal the associated Chern bands of THM.). Since BFPO is a layered compound with $\mathrm{Ba}$ layers residing between the $\mathrm{Fe}_{2}\left(\mathrm{PO}_{4}\right)_{2}$ layers, replacing $\mathrm{Ba}$ into other cations can be done by intercalation of substitute cations or by using thin-film growth technique.

Next, the results for LFPO and CFPO are presented. Structural optimizations within $R \overline{3}$ symmetry for both CFPO, KFPO, and LFPO with including van der Waals functionals were done to obtain the lattice parameters and internal coordinates, where the optimized structures are shown in Supplementary Materials. Note that, since results for CFPO and KFPO are almost identical to each other, except the c parameter, here we only show results from CFPO. Figure $3 \mathrm{~b} c$ shows the LFPO bulk and zigzag-edge bands, respectively, dominated by the $e_{\mathrm{g}}$ states $\left(U_{\text {eff }}=4 \mathrm{eV}\right)$. The occupied and unoccupied bands have Chern number $C=-1$ and +1 per a $\mathrm{Fe}_{2}\left(\mathrm{PO}_{4}\right)_{2}$ layer, respectively, with a well-defined bulk gap 
(a)

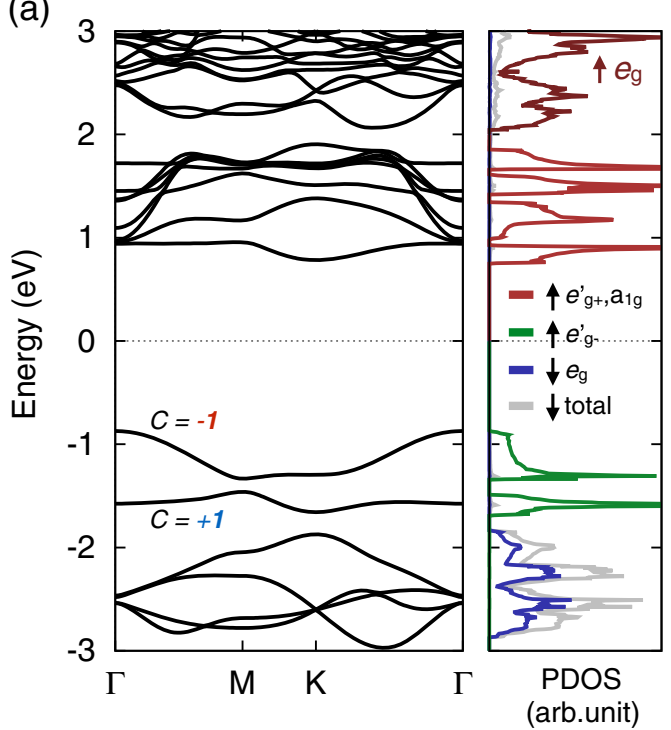

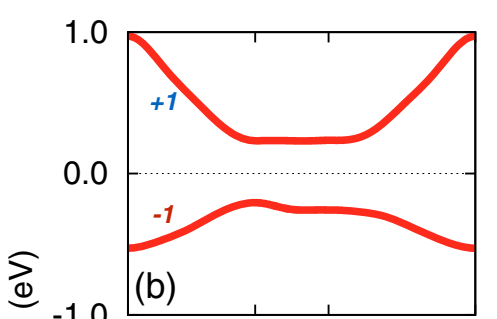

ํㅣㅁ

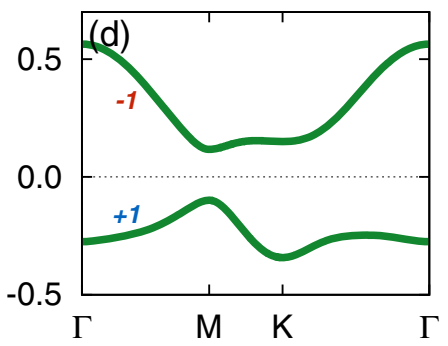

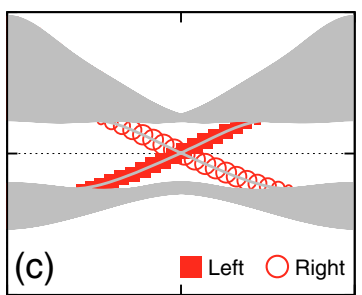

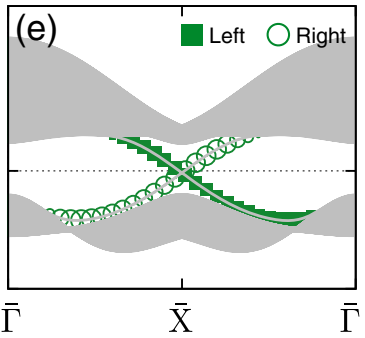

Fig. 3 a Band structure and projected density of states (PDOS) of single-layer BFPO (SL-BFPO), where Fe up spin bands (depicted as brown, dark red, and green lines in PDOS) are mostly shown above $-2 \mathrm{eV}$ with respect to the Fermi level. Chern numbers for four bands near the Fermi level are shown. Note that, the single-layer band structure is almost identical to the bulk band structure, except the position of Ba s-bands around $1 \sim 2 \mathrm{eV}$ above the Fermi level as shown in Supplementary Material. $U_{\text {eff }}=3 \mathrm{eV}$ is employed for Fe $3 d$ orbital. $\mathbf{b}$ Bulk $e^{\prime}{ }_{\mathrm{g}}$ bands of SLLFPO. The occupied and unoccupied $e^{\prime}{ }_{g \pm}$ bands are characterized by Chern number $C=-1$ and +1 per a Fe ${ }_{2}\left(\mathrm{PO}_{4}\right)_{2}$ layer, respectively. $\mathbf{c}$ Zigzag edge spectrum of LFPO, where the size of filled square and empty circular symbols depicting the edge weights. d, e show the bulk and edge bands of SL-CFPO, respectively, with prevailing $e^{\prime}{ }_{\mathrm{g}-}$ character. For LFPO and BFPO, $U_{\mathrm{eff}}=4 \mathrm{eV}$ is employed (color online)

Table 1. (Units in $\mathrm{meV}$ ) Hopping integrals for the $e^{\prime}{ }_{\mathrm{g} \pm}$ states in bulk LFPO and CFPO/KFPO, respectively, $e_{g_{+}}^{\prime}$-hopping integrals for SL-BFPO is shown for comparison ( $U_{\text {eff }}=4 \mathrm{eV}$ for all systems)

\begin{tabular}{llllll}
\hline & $t_{1}$ & $t_{2}$ & $t^{\prime}{ }_{2}$ & $t_{3}$ & $t_{\text {inter }}$ \\
\hline LFPO $\left(e^{\prime}{ }_{g^{+}}\right)$ & -210.4 & -50.3 & +22.0 & -15.7 & -5.2 \\
CFPO $\left(e^{\prime}{ }_{g_{-}}\right)$ & -138.5 & +46.2 & +21.4 & +5.3 & -0.2 \\
KFPO $\left(e^{\prime}{ }_{g_{-}}\right)$ & -137.6 & +46.3 & +24.0 & +3.8 & +1.2 \\
BFPO $\left(e^{\prime}{ }_{g_{-}}\right)$ & -116.7 & +24.0 & +39.1 & -7.7 & \\
\hline
\end{tabular}

$t_{3}$ and $t_{\text {inter }}$ are the third-nearest-neighbor and the largest interlayer hopping terms, respectively.

of 400 meV and showing a chiral edge state at one zigzag edge side as shown in Fig. 3c. On the other hand, Fig. 3d, e shows the CFPO bands, showing $e_{\mathrm{g}-}$ state character with opposite chirality. The bulk gap is about $220 \mathrm{meV}$, which is smaller than that of LFPO but still substantial. The hopping integrals for the $e_{\mathrm{g} \pm}$ states in both compounds are obtained from the Wannier orbital calculations, where the values are shown in Table 1. Note that they are well described by the THM. Unlike in $4 d$ - or $5 d$ honeycomb materials, such as $a-\mathrm{RuCl}_{3}$ or $A_{2} \mid \mathrm{IO}_{3}(A=\mathrm{Li}, \mathrm{Na})$ having the similar edge-sharing octahedral structure, AFPO show almost negligible third-nearest neighbor hopping terms due to the spatially localized $3 d$ orbitals. $^{31} t_{3}$ term in LFPO is larger than that of CFPO due to the smaller in-plane lattice constant, and further enhancing $t_{3}$ with epitaxial strain may induce a phase transition from $C= \pm 1$ to \pm 2 phase as reported in $a-\mathrm{RuCl}_{3}$ or $A_{2} \mathrm{IrO}_{3}$ (ref. 31).

It should be commented that, although the size of the BFPO band gap in DFT $+U$ and HSE results are well matched at $U_{\text {eff }} \simeq 5$ $\mathrm{eV}$, our results for all of $A$ FPO systems in this work remain robust in a wide range of $U_{\text {eff }}$ value, between 1.8 and $7 \mathrm{eV}$. In CFPO (KFPO) and LFPO, a bipartite charge ordering which breaks the topological phase is not observed across the $U_{\text {eff }}$ parameter range we tested. These observations suggest the robustness of THM and the resulting topological phase in these systems.

\section{DISCUSSION}

Our result suggests a new strategy in realizing THM phase with sizable gap in condensed matter systems. Previously, there have been roughly two different approaches; depositing magnetic ions or interfacing magnetic systems onto graphene or other honeycomb lattices, ${ }^{12,32,33}$ and planting magnetic ions in TIs. $^{11,18,19}$ We find that searching for systems with the ferromagnetic order in transition metal compounds with strong Hund's coupling and SOC is a promising way for the realization of THM. In addition to AFPO investigated in this study, there has been a report of possible quasi-2D Ising ferromagnets in several $3 d$-transition metal halides. ${ }^{34,} 35$ Since they have a $t_{2 \mathrm{~g}}$ orbital degree of freedom with spin splitting larger than the cubic crystal field, such system can show a similar atomic-orbital formation, which may possibly lead to the formation of THM. Heterostructures of transition metal oxides with magnetic ions, such as a double perovskite $\mathrm{Ba}_{2} \mathrm{FeReO}_{6}$ (ref. 36), can be another candidate.

Lastly, we comment on the size of the gap in BFPO. In the paramagnetic high-temperature $R \overline{3}$ phase, activation energy estimation from the conductivity is about $0.2 \mathrm{eV}$ (ref. 37), while optical spectroscopy estimates the gap size to be about $1.5 \mathrm{eV}$ (ref. 37), which are significantly smaller than our results of $\sim 3 \mathrm{eV}$ from the hybrid functional calculation. More reliable low-temperature data should be measured for further studies, and we emphasize that our results is robust independent of the value of $U$ and corresponding gap, as long as $U_{\text {eff }}$ is larger than $1.8 \mathrm{eV}$. A further theoretical studies incorporating quantum fluctuation effects for these systems when a fraction of electron or hole is added would be interesting, which may reveal fractional Chern insulator phases in these systems. ${ }^{38}$

In summary, we propose a way to search for realistic materials described by the THM: effectively spinless fermion with complex n.n. $\mathrm{n}$ hopping integrals can be found in ferromagnetic insulators with strong Hund's coupling and finite SOC. We apply this idea to a series of Fe-based honeycomb ferromagnetic oxides. We show that AFPO series is represented by THM, and predict that honeycomb CFPO, KFPO, and LFPO are candidates for the original THM exhibiting a 
quantum Hall effect without Landau levels. Our study provides a platform for exploring correlated topological materials, including possible fractional Chern insulators via strong correlations.

\section{METHODS}

DFT computations in this work are done with Vienna ab-initio Simulation Package (VASP) $)^{39}, 40$ and OPENMX codes. ${ }^{41}$ Especially structural optimization for C/KFPO and LFPOs with van der Waals functionals, and HSE hybrid functional calculations are done with using VASP, and in DFT $+U$ calculations and Wannier orbital calculations OPENMX was employed. DFT+ $U$ results from OPENMX and VASP are compared and found to be consistent with each other. For more computational details see Supplementary Material.

\section{ACKNOWLEDGEMENTS}

H.S.K. thanks Ji-Sang Park, Andrei Catuneanu, and Yige Chen for helpful comments. This work was supported by the NSERC of Canada and the center for Quantum Materials at the University of Toronto. Computations were mainly performed on the GPC supercomputer at the SciNet HPC Consortium. SciNet is funded by: the Canada Foundation for Innovation under the auspices of Compute Canada; the Government of Ontario; Ontario Research Fund-Research Excellence; and the University of Toronto.

\section{AUTHOR CONTRIBUTIONS}

Both author conceived and developed the core idea. H.S.K. performed ab-initio calculations and data analysis. Both authors contributed to discussion and writing the manuscript. H.Y.K. supervised the overall direction of the research.

\section{COMPETING INTERESTS}

The authors declare that they have no competing interests.

\section{REFERENCES}

1. Haldane, F. D. M. Model for a quantum hall effect without landau levels: Condensed-matter realization of the "parity anomaly". Phys. Rev. Lett. 61, 2015-2018 (1988).

2. Weng, H., Yu, R., Hu, X., Dai, X. \& Fang, Z. Quantum anomalous hall effect and related topological electronic states. Adv. Phys 64, 227-282 (2015).

3. Liu, C.-X., Zhang, S.-C. \& Qi, X.-L. The quantum anomalous hall effect: Theory and experiment. Annu. Rev. Condens. Matter Phys. 7, 301-321 (2016).

4. Ren, Y., Qiao, Z. \& Niu, Q. Topological phases in two-dimensional materials: a review. Rep. Prog. Phys. 79, 066501 (2016).

5. Kane, C. L. \& Mele, E. J. Quantum spin hall effect in graphene. Phys. Rev. Lett. 95 226801 (2005)

6. Kane, C. L. \& Mele, E. J. $Z_{2}$ topological order and the quantum spin hall effect. Phys. Rev. Lett 95, 146802 (2005).

7. Hasan, M. Z. \& Kane, C. L. Colloquium : Topological insulators. Rev. Mod. Phys. 82, 3045-3067 (2010).

8. Hsieh, D. et al. A topological Dirac insulator in a quantum spin Hall phase. Nature 452, 970-974 (2008).

9. Xia, Y. et al. Observation of a large-gap topological-insulator class with a single Dirac cone on the surface. Nat. Phys 5, 398-402 (2009).

10. Liu, C.-X., Qi, X.-L., Dai, X., Fang, Z. \& Zhang, S.-C. Quantum anomalous hall effect in $\mathrm{Hg}_{1-y} \mathrm{Mn}_{y} \mathrm{Te}$ quantum wells. Phys. Rev. Lett 101, 146802 (2008).

11. Yu, R. et al. Quantized anomalous hall effect in magnetic topological insulators. Science 329, 61-64 (2010).

12. Qiao, Z. et al. Quantum anomalous hall effect in graphene from rashba and exchange effects. Phys. Rev. B 82, 161414 (2010)

13. Zhang, H., Lazo, C., Blügel, S., Heinze, S. \& Mokrousov, Y. Electrically tunable quantum anomalous hall effect in graphene decorated by $5 d$ transition-metal adatoms. Phys. Rev. Lett. 108, 056802 (2012).

14. Xu, G., Lian, B. \& Zhang, S.-C. Intrinsic quantum anomalous hall effect in the kagome lattice $\mathrm{Cs}_{2} \mathrm{LiMn}_{3} \mathrm{~F}_{12}$. Phys. Rev. Lett. 115, 186802 (2015).

15. Guterding, D., Jeschke, H. O. \& Valentí, R. Prospect of quantum anomalous Hall and quantum spin Hall effect in doped kagome lattice Mott insulators. Sci. Rep. 6 25988 (2016).
16. Xiao, D., Zhu, W., Ran, Y., Nagaosa, N. \& Okamoto, S. Interface engineering of quantum Hall effects in digital transition metal oxide heterostructures. Nat Commun. 2, 596 (2011)

17. Cook, A. M. \& Paramekanti, A. Double perovskite heterostructures: Magnetism, chern bands, and chern insulators. Phys. Rev. Lett. 113, 077203 (2014).

18. Chang, C.-Z. et al. Experimental observation of the quantum anomalous hall effect in a magnetic topological insulator. Science 340, 167-170 (2013).

19. Chang, C.-Z. et al. High-precision realization of robust quantum anomalous Hall state in a hard ferromagnetic topological insulator. Nat. Mater. 14, 473-477 (2015).

20. Oka, T. \& Aoki, H. Photovoltaic hall effect in graphene. Phys. Rev. B 79, 081406 (2009).

21. Kitagawa, T., Oka, T., Brataas, A., Fu, L. \& Demler, E. Transport properties of nonequilibrium systems under the application of light: Photoinduced quantum hall insulators without landau levels. Phys. Rev. B 84, 235108 (2011).

22. Wang, Y. H., Steinberg, H., Jarillo-Herrero, P. \& Gedik, N. Observation of floquetbloch states on the surface of a topological insulator. Science 342, 453-457 (2013).

23. Mahmood, F. et al. Selective scattering between floquet-bloch and volkov states in a topological insulator. Nat. Phys 12, 306-310 (2016).

24. Jotzu, G. et al. Experimental realization of the topological Haldane model with ultracold fermions. Nature 515, 237-240 (2014).

25. Kabbour, $\mathrm{H}$. et al. A genuine two-dimensional ising ferromagnet with magnetically driven re-entrant transition. Angew. Chem. Int. Ed. 51, 11745-11749 (2012).

26. David, R. et al. Across the structural re-entrant transition in $\mathrm{BaFe}_{2}\left(\mathrm{PO}_{4}\right)_{2}$ : Influence of the two-dimensional ferromagnetism. J. Am. Chem. Soc. 135, 13023-13029 (2013).

27. Song, Y.-J., Lee, K.-W. \& Pickett, W. E. Large orbital moment and spin-orbit enabled Mott transition in the Ising Fe honeycomb lattice of $\mathrm{BaFe}_{2}\left(\mathrm{PO}_{4}\right)_{2}$. Phys. Rev. B 92, 125109 (2015)

28. Dudarev, S. L., Botton, G. A., Savrasov, S. Y., Humphreys, C. J. \& Sutton, A. P. Electron-energy-loss spectra and the structural stability of nickel oxide: An LSDA +U study. Phys. Rev. B 57, 1505-1509 (1998).

29. Heyd, J., Scuseria, G. E. \& Ernzerhof, M. Hybrid functionals based on a screened coulomb potential. J. Chem. Phys. 118, 8207-8215 (2003).

30. Song, Y.-J., Ahn, K.-H., Pickett, W. E. \& Lee, K.-W. Tuning ferromagnetic $\mathrm{BaFe}_{2}\left(\mathrm{PO}_{4}\right)_{2}$ through a high Chern number topological phase. Phys. Rev. B 94, 125134 (2016).

31. Catuneanu, A., Kim, H.-S., Can, O. \& Kee, H.-Y. Topological edge states in correlated honeycomb materials with strong spin-orbit coupling. Phys. Rev. B 94, 121118 (2016).

32. Qiao, Z. et al. Quantum anomalous hall effect in graphene proximity coupled to an antiferromagnetic insulator. Phys. Rev. Lett. 112, 116404 (2014).

33. Garrity, K. F. \& Vanderbilt, D. Chern insulators from heavy atoms on magnetic substrates. Phys. Rev. Lett. 110, 116802 (2013)

34. He, J., Ma, S., Lyu, P. \& Nachtigall, P. Unusual dirac half-metallicity with intrinsic ferromagnetism in vanadium trihalide monolayers. J. Mater. Chem. C 4, 2518-2526 (2016).

35. Liu, J., Sun, Q., Kawazoe, Y. \& Jena, P. Exfoliating biocompatible ferromagnetic crtrihalide monolayers. Phys. Chem. Chem. Phys. 18, 8777-8784 (2016).

36. Baidya, S., Waghmare, U. V., Paramekanti, A. \& Saha-Dasgupta, T. Hightemperature large-gap quantum anomalous hall insulating state in ultrathin double perovskite films. Phys. Rev. B 94, 155405 (2016).

37. David, R. et al. Reversible topochemical exsolution of iron in $\mathrm{BaFe}_{2}\left(\mathrm{PO}_{4}\right)_{2}$. Angew. Chem. Int. Ed 53, 13365-13370 (2014).

38. Neupert, T., Santos, L., Chamon, C. \& Mudry, C. Fractional quantum hall states at zero magnetic field. Phys. Rev. Lett. 106, 236804 (2011).

39. Kresse, G. \& Furthmüller, J. Efficient iterative schemes for $a b$ initio total-energy calculations using a plane-wave basis set. Phys. Rev. B 54, 11169-11186 (1996).

40. Kresse, G. \& Joubert, D. From ultrasoft pseudopotentials to the projector augmented-wave method. Phys. Rev. B 59, 1758-1775 (1999).

41. Ozaki, T. Variationally optimized atomic orbitals for large-scale electronic structures. Phys. Rev. B 67, 155108 (2003).

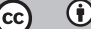

This work is licensed under a Creative Commons Attribution 4.0 International License. The images or other third party material in this article are included in the article's Creative Commons license, unless indicated otherwise in the credit line; if the material is not included under the Creative Commons license, users will need to obtain permission from the license holder to reproduce the material. To view a copy of this license, visit http://creativecommons.org/licenses/by/ $4.0 /$

(c) The Author(s) 2017

Supplementary Information accompanies the paper on the npj Quantum Materials website (doi:10.1038/s41535-017-0021-z). 